\title{
Red Ginseng Saponin Fraction A Isolated from Korean Red Ginseng by Ultrafiltration on the Porcine Coronary Artery
}

\author{
Young Hyun Jung ${ }^{1}$, Kwang Yeol Park ${ }^{1}$, Jin Hong Jeon ${ }^{1}$, Yi-Seong Kwak ${ }^{2}$, Yong-Bum Song ${ }^{2}$, \\ Jae-Joon Wee ${ }^{2}$, Man Hee Rhee ${ }^{1}$, and Tae Wan Kim ${ }^{1 *}$ \\ ${ }^{1}$ Department of Physiology, Kyungpook National University College of Veterinary Medicine, Daegu 702-701, Korea \\ ${ }^{2}$ Korea Ginseng Corporation Central Research Institute, Daejon 305-805, Korea
}

Red ginseng saponin fraction-A (RGSF-A) contains a high percentage of panaxadiol saponins that were isolated from Korean red ginseng by ultrafiltration. The aim of this study was to elucidate the effects of RGSF-A on the porcine distal left anterior descending (LAD) coronary artery. The relaxant responses to RGSF-A were examined during contractions induced by $100 \mathrm{nM} \mathrm{U} 46619$ (9,11-dideoxy-9a,11a-methanoepoxy-prostaglandin F2a), a stable analogue of thromboxane A2. RGSF-A dose-dependently induced biphasic (fast- and slow-) relaxation in the distal LAD coronary artery in the presence of an intact endothelium. The fast-relaxation was quickly achieved in a minute, and then the slow-relaxation was slowly developed and sustained for more than thirty minutes after the administration of RGSF-A. The slow-relaxation had a tendency to be bigger than the fast-relaxation. Fast relaxation induced by RGSF-A was almost blocked by $N_{\omega}$-Nitro-L-arginine methyl ester (L-NAME), a nitric oxide synthase synthase inhibitor and 1H-[1,2,4] oxadiazolo[4,3-a]quinoxalin-1-one (ODQ), a guanylate cyclase inhibitor. However slow relaxation induced by RGSF-A was only partially inhibited by L-NAME and ODQ. In the endothelium-removed ring, RGSF-A evoked only slowrelaxation to a certain extent. These data suggest that RGSF-A induced both endothelium dependent fast- and slow-relaxation and endothelium independent slow-relaxation in the porcine distal LAD coronary artery. The endothelium dependent fast-relaxation is mediated by the nitric oxide (NO)-cGMP pathway, and the endothelium dependent slow-relaxation is at least partially mediated by the NO-cGMP pathway. However, the endothelium-independent slow-relaxation remains to be elucidated.

Keywords: Ginseng, Red ginseng saponin fraction-A, Porcine coronary artery, Endothelium, Nitric oxide

\section{INTRODUCTION}

Korean red ginseng (Panax ginseng) has been used for more than two thousand years in traditional medicine in the Far Eastern Asian regions for a variety of disorders. Ginsenosides, which have a four ring steroid-like structure with attached sugar moieties, are considered a biologically active component of $P$. ginseng [1-3]. The ginseng root contains more than 30 types of ginsenosides divided into two major groups based on their chemical

(c) This is an Open Access article distributed under the terms of the Creative Commons Attribution Non-Commercial License (http://creativecommons.org/licenses/by-nc/3.0/) which permits unrestricted non-commercial use, distribution, and reproduction in any medium, provided the original work is properly cited. structure: panaxadiols with sugar moieties at the C-3 and C-21 positions of the sterol structure and panaxatriols with sugar moieties at positions C-6 and C-21 [4]. Ginsenoside $\mathrm{Rb}_{1}, \mathrm{Rb}_{2}, \mathrm{Rb}_{3}, \mathrm{Rc}, \mathrm{Rd}, \mathrm{Rg}_{3}, \mathrm{Rh}_{2}$ and $\mathrm{Rs}_{1}$ represent the panaxadiols, whereas ginsenoside $\mathrm{Re}, \mathrm{Rf}, \mathrm{Rg}_{1}$, $\mathrm{Rg}_{2}$ and $\mathrm{Rh}_{1}$ represent panaxatriols. It has been reported that panaxadiol and panaxatriol have different effects in various tissue [5-8].

Received 26 Nov. 2010, Revised 08 Dec. 2010, Accepted 09 Dec. 2010

*Corresponding author

E-mail: twkim@mail.knu.ac.kr

Tel: +82-53-950-7791, Fax: +82-53-950-5955 
It has been reported that cardiovascular protection effects of the ginseng root and ginsenosides are closely associated with vasodilation and promotion of endothelium-derived nitric oxide which enhances the accumulation of cGMP [9-13]. However, these results on vasodilation induced by ginseng were mainly obtained from experiments using aortic rings in vitro. Although there are several reports indicating that ginseng or ginsenosides can dilate the coronary artery of the heart [1418], there are few reports that state the effects of ginseng or ginsenosides on the coronary artery are mediated by the nitric oxide (NO) pathway [19]. The aim of this study was to investigate whether ginsenosides relax the porcine coronary artery by the NO pathway in vitro. In the present study, we used red ginseng saponin fraction A (RGSF-A) as the ginsenoside mixture.

\section{MATERIALS AND METHODS}

\section{Materials}

RGSF-A is a fraction containing a high percentage of panaxadiols, which was isolated from Korean red ginseng by ultrafiltration. RGSF-A was kindly obtained from the Korea Ginseng Corporation (Daejeon, Korea). Nine ginsenosides, i.e., $\mathrm{Rb}_{1}, \mathrm{Rb}_{2}, \mathrm{Rc}, \mathrm{Rd}, \mathrm{Re}, \mathrm{Rf}, \mathrm{Rg}_{1}$, $\mathrm{Rg}_{2}$ and $\mathrm{Rg}_{3}$ were identified from RGSF-A through a comparison of the retention times with authentic compounds. The contents of the ginsenosides were 9.94, 4.22, $4.29,1.72,1.66,1.27,1.52,0.695$ and $0.695 \%$ of the dry extract, respectively (Table 1). The following compounds were used: U46619 (Cayman, Ann Arbor, MI, USA), sodium nitroprusside (Sigma, St. Louis, MO, USA), N $\omega$ Nitro-L-arginine methyl ester (L-NAME, Sigma) and 1H-[1,2,4] oxadiazolo[4,3-a]quinoxalin-1-one (ODQ; Calbiochem-Novabiochem, La Jolla, CA, USA). ODQ was prepared in dimethyl sulfoxide. The other compounds were prepared in distilled water. Further dilutions to the desired concentrations were made with physiological salt solution (PSS).

\section{Preparation}

Porcine hearts were obtained from a local slaughterhouse and transported in ice-cold oxygenated PSS. The composition of PSS was as follows (in $\mathrm{mM}$ ): $\mathrm{NaCl} 118$, $\mathrm{KCl} 4.7, \mathrm{MgCl}_{2}$ 1.2, $\mathrm{CaCl}_{2} 2.5, \mathrm{KH}_{2} \mathrm{PO}_{4} 1.2, \mathrm{NaHCO}_{3}$ 25.0 and glucose 10.0. The distal part of the left anterior descending (LAD) coronary artery (outer diameter, 0.8$1.5 \mathrm{~mm}$ ) was dissected from the heart in oxygenated PSS. The isolated artery was trimmed of fat and connective tissues under a dissecting microscope and cut into rings, $4 \mathrm{~mm}$ in length. Care was taken to ensure that the endothelium was not damaged during the processing of the tissue preparation. Where indicated, the endothelial cells were removed by gently rubbing the inner surface of the vessel with a moistened cotton thread moistened with PSS. The endothelium-removed rings were confirmed if substance $\mathrm{P}(10 \mathrm{nM})$ did not induce relaxation. Substance $P$ induces endothelium-dependent vasorelaxation in the porcine coronary artery [20].

\section{Measurement of isometric tension}

The arterial ring was suspended by a pair of stainless steel stirrups in a water-jacketed bath filled with $10 \mathrm{~mL}$ of PSS. The solution in the bath was gassed with $95 \%$ $\mathrm{O}_{2}$ and $5 \% \mathrm{CO}_{2}$, and its temperature was maintained at $37^{\circ} \mathrm{C}$. The upper end of the strip was connected to the isometric force transducer (FT-03; Grass-Telefactor, West Warwick, RI, USA). The output of the transducer was processed through Powerlab 2/25 and Chart 5.2 (AD Instruments, Castlehill, Australia). The ring was stretched until an optimal tension of $2 \mathrm{~g}$ was loaded and then allowed to equilibrate for at least $60 \mathrm{~min}$ before the start of the experiments. The RGSF-A or other drugs were administered after the contraction had reached a plateau level by U46619 (100 nM , 9,11-dideoxy-9a,11amethanoepoxy-prostaglandin F2a), a stable analogue of thromboxane $\mathrm{A}_{2}$. Rings that failed to produce a contraction greater than $3 \mathrm{~g}$ with $\mathrm{U} 46619$ or relaxed by less than $50 \%$ with substance $\mathrm{P}(5 \mathrm{nM})$ were discarded except when using the endothelium-removed rings.

\section{Statistical analysis}

All values were expressed as the mean \pm SEM. Statistical assessment of the data was calculated made by student's $t$-test. A $p$-value of less than 0.05 was taken to be statistically significant.

Table 1. Percentage of ginsenosides present in RGSF-A

\begin{tabular}{ccccccccccccccc}
\hline \multicolumn{11}{c}{ Composition of RGSF-A (dry extract, \%) } \\
\hline $\mathrm{Rb}_{1}$ & $\mathrm{Rb}_{2}$ & $\mathrm{Rc}$ & $\mathrm{Rd}$ & $\mathrm{Re}$ & $\mathrm{Rf}$ & $\mathrm{Rg}_{1}$ & $\mathrm{Rg}_{2}$ & $\mathrm{Rg}_{3}$ & Total & Diol & $\mathrm{Triol}$ & $\mathrm{PD} / \mathrm{PT}$ \\
9.94 & 4.22 & 4.29 & 1.72 & 1.66 & 1.27 & 1.52 & 0.695 & 0.925 & 26.24 & 21.10 & 5.15 & 4.10 \\
\hline
\end{tabular}

RGSF-A, red ginseng saponin fraction-A; PD/PT, panaxadiol/panaxatriol. 


\section{RESULTS}

\section{Red ginseng saponin fraction-A induced biphasic relaxation}

RGSF-A was administered into the bath in a cumulative method $(1-300 \mu \mathrm{g} / \mathrm{mL})$ when contraction of the

A

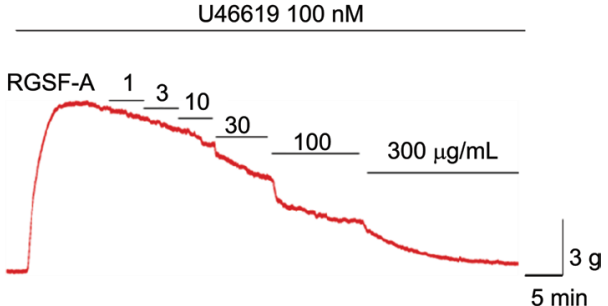

B

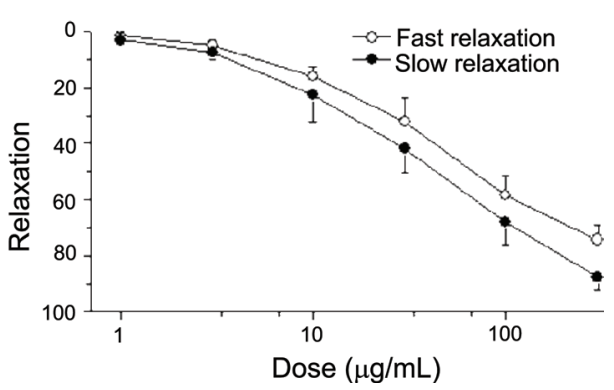

Fig. 1. Red ginseng saponin fraction-A (RGSF-A) dose-dependently induced biphasic (fast- and slow-) relaxation in the distal left anterior descending coronary artery with the endothelium contracted by U46619. The fast-relaxation was quickly achieved in a minute, and then the slow-relaxation was slowly developed and sustained for more than thirty minutes after the administration of RGSF-A (A). The slow-relaxation had a tendency to be bigger than the fast-relaxation (B) $(n=6)$. porcine distal LAD coronary artery by $\mathrm{U} 46619(100 \mathrm{nM})$ reached the plateau. RGSF-A dose-dependently induced biphasic (fast- and slow-) relaxation in the distal LAD coronary artery in the presence of intact endothelium. Fast-relaxation was quickly achieved in a minute, and then the slow-relaxation was slowly developed and sustained for more than thirty minutes after administration of RGSF-A (Fig. 1A). The percentage of fast-relaxation of RGSF on the swine distal LAD coronary artery was $1.4 \pm 1.11,4.9 \pm 2.19,15.9 \pm 3.65,32.1 \pm 8.48,58.3 \pm 6.79$ and $74.3 \pm 5.43 \%$ at doses of $1,3,10,30,100$ and $300 \mu \mathrm{g} / \mathrm{mL}$, respectively. In addion, the percentage of slow-relaxation of RGSF on swine distal LAD coronary artery was $3.2 \pm 1.15,7.34 \pm 2.47,22.6 \pm 9.44,41.90 \pm 8.69,67.8 \pm 8.05$ and $87.8 \pm 4.40 \%$ at doses of $1,3,10,30,100$ and $300 \mu \mathrm{g} /$ $\mathrm{mL}$, respectively. The $\mathrm{IC}_{50}$ of RGSF-A in fast- and slowrelaxation were 43.3 and $64.4 \mu \mathrm{g} / \mathrm{mL}$, respectively. The slow-relaxation had a tendency to be bigger than the fastrelaxation (Fig. 1B).

\section{Involvement of NO pathway in RGSF-A induced va- sorelaxation}

Administration of RGSF-A $(100 \mu \mathrm{M})$ alone evoked fast- and slow- relaxation in the porcine distal LAD coronary artery (Fig. 2A). The slow relaxation was much bigger than the fast-relaxation (Figs. 2A and 3A). With the pretreatment of L-NAME $(100 \mu \mathrm{M})$, a nitric oxide synthase inhibitor, the fast-relaxation by RGSF-A was almost abolished, and the slow- relaxation by RGSF-A was significantly attenuated by L-NAME (Fig. 2B, C). However, with the pre-incubation of L-NAME, the RGSF-
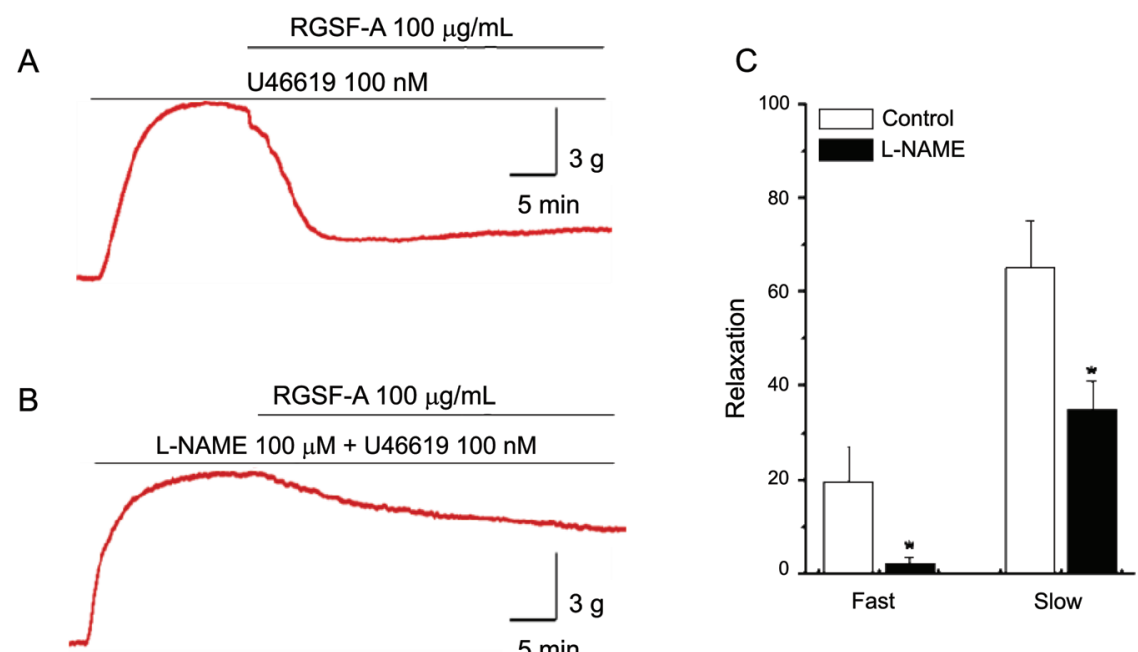

Fig. 2. Effects of red ginseng saponin fraction-A (RGSF-A) on the porcine coronary artery were inhibited by N $\omega$-Nitro-L-arginine methyl ester (LNAME). Administration of RGSF-A $(100 \mu \mathrm{g} / \mathrm{mL})$ induced fast- and slow- relaxation in the porcine distal left anterior descending coronary artery (A). Fast-relaxation by RGSF-A was almost abolished by L-NAME $(100 \mu \mathrm{M})$ and slow- relaxation by RGSF-A was significantly attenuated by L-NAME $(B, C)$. Both tracings were recorded from the same tissue $(n=5, " p<0.05$ vs. control). 


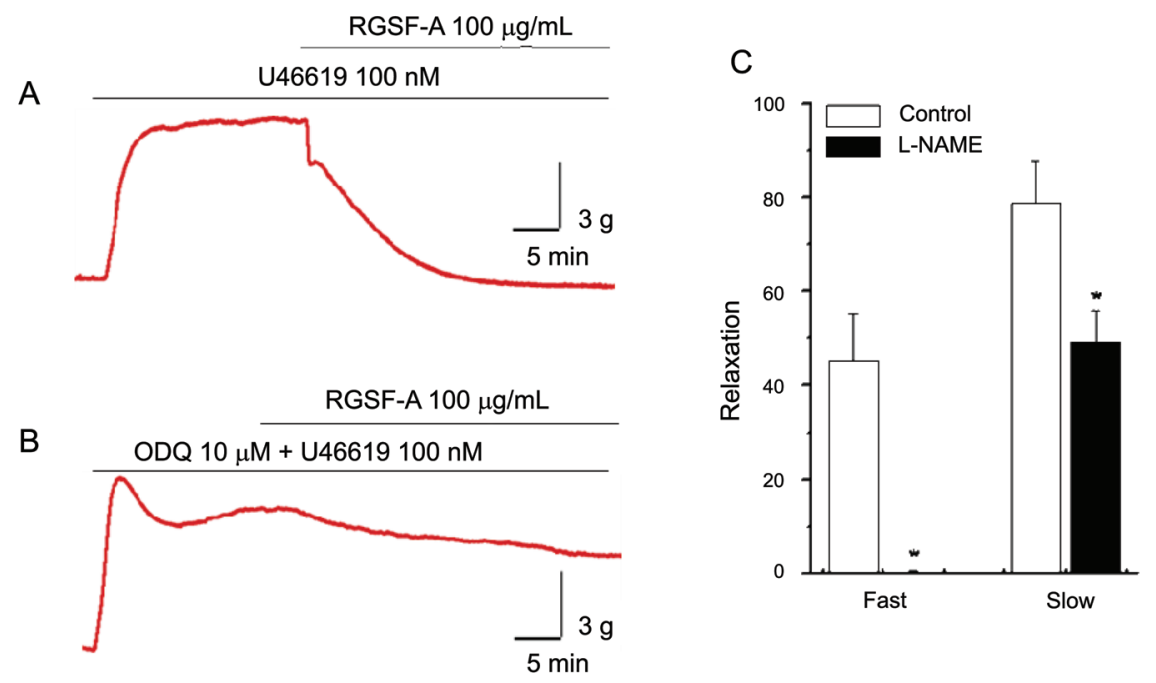

Fig. 3. Effects of red ginseng saponin fraction-A (RGSF-A) on the porcine coronary artery were inhibited by $1 \mathrm{H}-[1,2,4]$ oxadiazolo[4,3-a]quinoxalin-1-one (ODQ). Fast- and slow- relaxation were induced by RGSF-A in the distal left anterior descending coronary artery (A). Fast- relaxation evoked by RGSF-A was almost abolished by ODQ $(10 \mu \mathrm{M})$ and the slow-relaxation was significantly attenuated by ODQ $(\mathrm{B}, \mathrm{C})$. Both tracings were recorded from the same tissue $\left(n=5,{ }^{*} p<0.05\right)$.

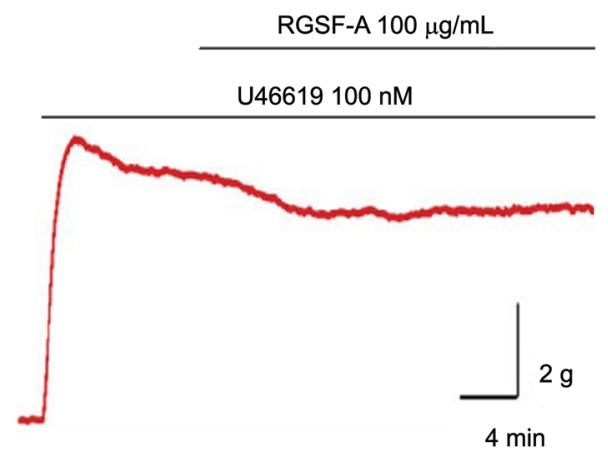

Fig. 4. Effects of red ginseng saponin fraction-A (RGSF-A) on the porcine distal left anterior descending (LAD) coronary artery without an endothelium. RGSF-A induced slow relaxation to a small degree but did not evoke fast relaxation in the distal LAD coronary artery without an endothelium.

A induced slow-relaxation still remained to a degree. The effects of RGSF-A in the absence or presence of LNAME were recorded in the same rings. ODQ, soluble guanylate cyclase inhibitor, also inhibited the effects of RGSF-A on the distal LAD coronary artery (Fig. 3B). Fast-relaxation by RGSF-A was completely abolished and slow-relaxation by RGSF-A was significantly attenuated by ODQ $(10 \mu \mathrm{M})$. However, slow-relaxation by RGSF-A still remained to a degree in the pretreatment of ODQ (Fig. 3B, C).

\section{Effects of RGSF-A on an endothelium-removed coronary artery ring}

Administration of RGSF-A induced a slowly devel- oped relaxation in the endothelium-removed coronary artery ring. Typical tracings indicated that RGSF-A evoked a slow relaxation to a certain extent but not a fast-relaxation in an endothelium-denuded coronary artery (Fig. 4).

\section{DISCUSSION}

In this study, RGSF-A dose-dependently induced biphasic (fast- and slow-) relaxation in the porcine distal LAD coronary artery. RGSF-A is the fraction containing a high percentage of panaxadiols, which was isolated from the Korean red ginseng by ultrafiltration. Fast-relaxation was quickly achieved in a minute, and then slowrelaxation was slowly developed and sustained for more than thirty minutes after the administration of RGSF-A. In a cumulative addition of RGSF-A, the extent of fastrelaxation was close to that of the slow-relaxation. However, a single administration of RGSF-A induced a much bigger slow-relaxation than fast-relaxation in porcine distal LAD coronary artery. We think that the small difference between the fast- and slow-relaxation in a cumulative addition of RGSF-A is due to the administration of higher concentrations of RGSF-A after slow relaxation has developed by the previously added low concentrations of RGSF-A.

Recently, it was reported that total ginsenosides have cardioprotective effects and can enhance the coronary artery flow against ischemia/reperfusion injury in isolated rat hearts $[6,19]$. This increasing effect of total ginsen- 
osides on coronary artery flow is mediated, at least partially, by the NO pathway. The relaxing effect of ginsenosides on the coronary artery has been reported in rabbits [15] and swine [14,21] in vitro. However, these reports did not consider whether the effect of the ginsenosides on the coronary artery was mediated by NO.

In this experiment, the RGSF-A induced fast-relaxation was almost blocked by L-NAME (nitric oxide synthase inhibitor) and completely blocked by ODQ (sGC inhibitor) in the porcine distal LAD coronary artery with an intact endothelium. In addition, the slow-relaxation was significantly attenuated by L-NAME and ODQ. In the endothelium-removed rings, RGSF-A induced only a slow-relaxation to a certain extent without any fastrelaxation. These data suggest that the fast-relaxation induced by RGSF-A is mediated by the NO-cGMP pathway and is endothelium-dependent. The slow-relaxation is partially mediated by the endothelium-dependent NO-cGMP pathway. However, these data could not exclude the possibility that the slow-relaxation induced by RGSF-A is also involves another endothelium-dependent mechanism like as endothelium-derived hyperpolarizing factor $[19,22]$.

In the present study, our data suggest that the slow relaxation induced by RGSF-A consists of both endothelium-dependent and independent mechanisms, and currently, our data cannot explain the mechanism for the endothelium-independent slow relaxation induced by RGSF-A. It was reported that ginsenosides induced endothelium-independent relaxation in the swine coronary artery [21,23] and rat aortic rings [24]. Increasing data show that ginsenosides have effects on the ion channels of vascular smooth muscle cells. It was reported that ginsenosides activate large-conductance $\mathrm{Ca}^{2+}$-activated $\mathrm{K}^{+}$channels in rabbits [15] and rat aortic artery smooth muscle cells [25]. On other hand, it is known that ginsenosides inhibit L-type $\mathrm{Ca}^{2+}$-current in porcine coronary artery smooth muscle cells [21]. These data might explain the endothelium-independent relaxation of RGSF-A.

In conclusion, RGSF-A induced both endothelium dependent fast- and slow-relaxation and endothelium independent slow-relaxation in the porcine distal LAD coronary artery. The endothelium dependent fast-relaxation is mediated by the NO-cGMP pathway and the endothelium dependent slow-relaxation is at least partially mediated by the NO-cGMP pathway. However, the endothelium-independent slow-relaxation remains to be elucidated.

\section{REFERENCES}

1. Attele AS, Wu JA, Yuan CS. Ginseng pharmacology: multiple constituents and multiple actions. Biochem Pharmacol 1999;58:1685-1693.

2. Furukawa T, Bai CX, Kaihara A, Ozaki E, Kawano T, Nakaya Y, Awais M, Sato M, Umezawa Y, Kurokawa J. Ginsenoside Re, a main phytosterol of Panax ginseng, activates cardiac potassium channels via a nongenomic pathway of sex hormones. Mol Pharmacol 2006;70:19161924.

3. Cho JG, Lee MK, Lee JW, Park HJ, Lee DY, Lee YH, Yang DC, Baek N. Physicochemical characterization and NMR assignments of ginsenosides $\mathrm{Rb}_{1}, \mathrm{Rb}_{2}, \mathrm{Rc}$, and $\mathrm{Rd}$ isolated from Panax ginseng. J Ginseng Res 2010;34:113121.

4. Kaku T, Miyata T, Uruno T, Sako I, Kinoshita A. Chemico-pharmacological studies on saponins of Panax ginseng C. A. Meyer. II. Pharmacological part. Arzneimittelforschung 1975;25:539-547.

5. Chang MS, Lee SG, Rho HM. Transcriptional activation of $\mathrm{Cu} / \mathrm{Zn}$ superoxide dismutase and catalase genes by panaxadiol ginsenosides extracted from Panax ginseng. Phytother Res 1999;13:641-644.

6. Kim TH, Lee SM. The effects of ginseng total saponin, panaxadiol and panaxatriol on ischemia/reperfusion injury in isolated rat heart. Food Chem Toxicol 2010;48:15161520 .

7. Kudo K, Tachikawa E, Kashimoto T, Takahashi E. Properties of ginseng saponin inhibition of catecholamine secretion in bovine adrenal chromaffin cells. Eur J Pharmacol 1998;341:139-144.

8. Lee HJ, Kim SR, Kim JC, Kang CM, Lee YS, Jo SK, Kim TH, Jang JS, Nah SY, Kim SH. In Vivo radioprotective effect of Panax ginseng C.A. Meyer and identification of active ginsenosides. Phytother Res 2006;20:392395.

9. Chen X, Gillis CN, Moalli R. Vascular effects of ginsenosides in vitro. Br J Pharmacol 1984;82:485-491.

10. Chen X. Cardiovascular protection by ginsenosides and their nitric oxide releasing action. Clin Exp Pharmacol Physiol 1996;23:728-732.

11. Gillis CN. Panax ginseng pharmacology: a nitric oxide link? Biochem Pharmacol 1997;54:1-8.

12. Kim ND, Kang SY, Schini VB. Ginsenosides evoke endothelium-dependent vascular relaxation in rat aorta. Gen Pharmacol 1994;25:1071-1077.

13. Hur MH, Lee MS, Yang HJ, Kim C, Bae IL, Ernst E. Ginseng for reducing the blood pressure in patients with hypertension: a systematic review and meta-analysis. J 
Ginseng Res 2010;34:342-347.

14. Chang SJ, Suh JS, Jeon BH, Nam KY, Park HK. Vasorelaxing effect by protopanaxatriol and protopanaxadiol of Panax ginseng in the pig coronary artery. Korean J Ginseng Sci 1994;18:95-101.

15. Chung I, Kim ND. Ginseng saponins enhance maxi $\mathrm{Ca}^{2+}+$ activated $\mathrm{K}+$ currents of the rabbit coronary artery smooth muscle cells. J Ginseng Res 1999;23:230-234.

16. Kang SY, Kim SH, Schini VB, Kim ND. Dietary ginsenosides improve endothelium-dependent relaxation in the thoracic aorta of hypercholesterolemic rabbit. Gen Pharmacol 1995;26:483-487.

17. Kim JY, Kim YC, Lee MG, Kwon JW, Yoo M. Effects of water deprivation on the pharmacokinetics of DA-8159, a new erectogenic, in rats. J Pharm Pharm Sci 2006;9:1021.

18. Chung IM, Lim JW, Pyun WB, Kim HY. Korean red ginseng improves vascular stiffness in patients with coronary artery disease. J Ginseng Res 2010;34:212-218.

19. Yi XQ, Li T, Wang JR, Wong VK, Luo P, Wong IY, Jiang $\mathrm{ZH}$, Liu L, Zhou H. Total ginsenosides increase coronary perfusion flow in isolated rat hearts through activation of PI3K/Akt-eNOS signaling. Phytomedicine 2010;17:10061015.

20. Matsumoto T, Kinoshita M, Toda N. Mechanisms of endothelium-dependent responses to vasoactive agents in isolated porcine coronary arteries. J Cardiovasc Pharmacol 1993;21:228-234.

21. Kim JH, Lee JH, Jeong SM, Lee BH, Yoon IS, Lee JH, Choi SH, Kim DH, Park TK, Kim BK et al. Stereospecific effects of ginsenoside $\mathrm{Rg}_{3}$ epimers on swine coronary artery contractions. Biol Pharm Bull 2006;29:365-370.

22. Yang Q, Zhang RZ, Yim AP, He GW. Release of nitric oxide and endothelium-derived hyperpolarizing factor (EDHF) in porcine coronary arteries exposed to hyperkalemia: effect of nicorandil. Ann Thorac Surg 2005;79:2065-2071.

23. Kim HB, Kang CW, Kim BS, Kwon JK, Yu IJ, Roh YS, Nah SY, Ejaz S, Kim JH. Beneficial role of ginseng saponin on hemodynamic functions of porcine blood vessel. J Ginseng Res 2010;34:314-320.

24. Kim ND, Kang SY, Kim MJ, Park JH, Schini-Kerth VB. The ginsenoside $\mathrm{Rg}_{3}$ evokes endothelium-independent relaxation in rat aortic rings: role of $\mathrm{K}+$ channels. Eur $\mathrm{J}$ Pharmacol 1999;367:51-57.

25. Nakaya Y, Mawatari K, Takahashi A, Harada N, Hata A, Yasui S. The phytoestrogen ginsensoside Re activates potassium channels of vascular smooth muscle cells through PI3K/Akt and nitric oxide pathways. J Med Invest 2007;54:381-384. 